\title{
Defect distributions in thin film solar cells deduced from admittance measurements under different bias voltages
}

\author{
Koen Decock, ${ }^{1, \text { a) }}$ Samira Khelifi, ${ }^{1}$ Stephan Buecheler, ${ }^{2}$ Fabian Pianezzi, ${ }^{2}$ \\ Ayodhya N. Tiwari, ${ }^{2}$ and Marc Burgelman ${ }^{1}$ \\ ${ }^{1}$ Department of Electronics and Information Systems (ELIS), Universiteit Gent, St-Pietersnieuwstraat 41, \\ B-9000 Gent, Belgium \\ ${ }^{2}$ Laboratory for Thin Films and Photovoltaics, EMPA, Überlandstrasse 129, CH-8600 Dübendorf, Switzerland
}

(Received 21 December 2010; accepted 18 August 2011; published online 28 September 2011)

\begin{abstract}
The voltage dependence of the derivative of the capacitance to (the logarithm of) the measurement frequency is investigated. Relations describing this dependence are derived for the influence of carrier freeze out, of a defect distribution, and of a back contact barrier. The validity of these relations is investigated with numerical simulations.

Considering the extraction of the defect density from capacitance-frequency measurements, the extension of existing formulas to different bias voltages leads to an improved accuracy and the possibility to investigate spatial non-uniformities while preserving a direct link between the defect level energy and the apparent defect density. This is illustrated with voltage dependent admittance measurements of thin film $\mathrm{Cu}(\mathrm{In}, \mathrm{Ga}) \mathrm{Se}_{2}$-based solar cell devices. (C) 2011 American Institute of Physics. [doi:10.1063/1.3641987]
\end{abstract}

\section{INTRODUCTION}

Thin film solar cells have achieved efficiencies of up to $20 \%{ }^{1}$ Despite these excellent results, the understanding of the underlying mechanisms and the influence of defects on its performance is still incomplete. The determination of the energetic position of the defects and of their density of states is important and can be performed with several techniques such as deep level transient spectroscopy, photoluminescence, capacitance-voltage $(C-V)$ measurements, drive level capacitance profiling (DLCP), or admittance spectroscopy (AS). ${ }^{2,3}$ In thin film solar cells defect level distributions are often present rather than discrete defects, which complicates the application of these techniques. Walter et al. ${ }^{4}$ developed a technique to determine defect energy distributions from admittance measurements under zero bias conditions, by calculating the derivative of the capacitance to the frequency.

As defect distributions in polycrystalline materials are often metastable and hence, bias induced, ${ }^{5-7}$ it is important to be able to perform measurements under different bias conditions. Thin film solar cells do not always have a uniform composition, thus several properties vary throughout the structure, either intentionally or not. Since capacitance techniques can only detect defects at the intersection of the Fermi level with the defect level, it is interesting to shift the Fermi levels by applying a bias in order to be able to detect defects at various positions in the structure.

Since there are several possible origins of admittance, ${ }^{8}$ there has always been some controversy about the interpretation of admittance measurements., ${ }^{9,10}$ Performing measurements under different bias conditions can provide extra input which could allow us to make the distinction between several possible origins of an admittance feature which is encountered.

\footnotetext{
${ }^{\text {a) }}$ Author to whom correspondence should be addressed. Electronic mail: Koen.Decock@elis.ugent.be.
}

In this article the voltage dependence of the derivative of the capacitance to the angular frequency is investigated. Three processes contributing to the capacitance are assessed: the influence of carrier freeze out, of charging and discharging defect distributions, and of a (back contact) barrier. In order to assess the influence of a defect distribution, the theory developed by Walter $e t$ al., ${ }^{4}$ has been extended so that it can be applied when an external bias is present.

The reliability of the calculated voltage dependencies is validated with numerical modeling on different structures using the solar cell device simulator, SCAPS ${ }^{11}$ (version 2.9). ${ }^{12}$ The voltage dependencies are also applied to the admittance measurements of thin film $\mathrm{Cu}(\mathrm{In}, \mathrm{Ga}) \mathrm{Se}_{2}$-based solar cell devices, illustrating the potential of voltage dependent admittance spectroscopy to obtain additional information about defect density profiles and the origin of the capacitance steps in capacitance frequency measurements.

\section{Theory}

Admittance measurements on solar cells are commonly interpreted as if the admittance, $Y(\omega)$, consists of a parallel connection of a capacitance, $C(\omega)$, and a conductance, $G(\omega)$,

$$
Y(\omega)=\frac{\tilde{J}}{\tilde{u}_{e x t}}=j \omega C(\omega)+G(\omega),
$$

with $\tilde{J}$ being the ac current and $\tilde{u}_{\text {ext }}$ the external applied ac voltage. The analysis of the admittance as a function of the angular frequency, $\omega$, temperature, $T$, and external applied voltage, $V$, can yield valuable information about the cell structure. According to the Kramers-Kronig relations the analysis of $C$ and $G / \omega$ is equivalent, and in this paper we will focus on the analysis of the capacitance.

There are several processes which can contribute to the capacitance, provided these processes can follow the applied 
ac-signal. When measuring the capacitance while varying the frequency so that a process can contribute to the capacitance at low frequency but no longer at high frequency, a step in the capacitance will be seen. The voltage dependence of the inflection frequency at which this step occurs and the derivative of the capacitance at this frequency will be investigated.

\section{Contribution of majority charge carriers}

Provided that the majority carrier can respond to the ac signal, the capacitance is determined by the space charge region width. Therefore, the angular frequency should be smaller than the dielectric relaxation frequency, $\omega_{D}$,

$$
\omega_{D}=\frac{\sigma}{\varepsilon},
$$

with $\sigma$ being the conductivity and $\varepsilon$ the dielectric permittivity. The conductivity is temperature dependent since it is proportional to the mobility $(\mu)$ and the carrier density. Hence, $\omega_{\mathrm{D}}$ is usually not in the frequency range commonly measured in admittance spectroscopy except at very low temperatures. ${ }^{9,13}$ However, when assuming hopping conduction, a capacitance step at higher temperatures can be interpreted as mobility freeze out. ${ }^{14}$

Li et al., ${ }^{13}$ calculated the voltage dependence of a capacitance step due to carrier freeze out in combination with the quasi-neutral region. The inflection frequency and derivative of the capacitance at this frequency is given by,

$$
\begin{aligned}
\omega_{0} & =\frac{w}{t} \frac{\sigma}{\varepsilon},\left.\omega \frac{d C}{d \omega}\right|_{\omega=\omega_{0}} \\
& =\frac{\varepsilon A}{2}\left(\frac{1}{t}-\frac{1}{w}\right),
\end{aligned}
$$

with $t$ being the absorber thickness, $w$ the depletion width, and $A$ the sample area. Here, $\omega_{0}$ is proportional to $\left(V_{b i}-V\right)^{1 / 2}$. If the absorber thickness can be considered much larger than the depletion width the derivative of the capacitance is proportional to $1 / w$ and thus, to $\left(V_{b i}-V\right)^{-1 / 2}$. Otherwise, when the absorber is almost entirely depleted, it is voltage independent. The inflection frequency is temperature dependent. Assuming the majority carriers to be holes, the conductance in Eq. (3) can be rewritten as, $\sigma=q \mu_{h} p=q \mu_{h} N_{V} \exp \left[\left(E_{V}-E_{F p}\right) / k T\right]$, leading to the following expression for the activation energy:

$$
E_{\omega}=E_{F p}-E_{V}=k T \ln \left(\frac{w}{t} \frac{\mu_{h} q N_{V}}{\varepsilon \omega_{0}}\right)=k T \ln \left(\frac{\xi_{0} T^{m}}{\omega_{0}}\right),
$$

where $T$ represents the temperature, $k$ is Boltzmann's constant, and $q$ is the elementary charge; $E_{C}$ and $E_{V}$ are the energy of the conduction and valence band edge, respectively, $N_{C}$ and $N_{V}$ are the effective density of states in the bands, and $E_{F n, F p}$ represent the quasi Fermi level of electrons and holes, respectively. The activation energy, $E_{\omega}$, can be determined by making an appropriate Arrhenius diagram $\left(\omega_{0} / T^{m}\right.$ versus $\left.1 / T\right)$. The exponent, $m$, is determined by the temperature dependence of the mobility, $\mu_{h}$, and the effective density of states, $N_{V}$. If the mobility is assumed to be temperature independent, this exponent equals $3 / 2$.

\section{Contribution of defect levels}

The charging and discharging of defects can also give a contribution to the capacitance. Walter et al. ${ }^{4}$ developed a procedure to investigate the effect of defect levels on the derivative of the capacitance under thermal equilibrium conditions. This theory is extended here so that it can be applied to different bias conditions as well.

The electron occupation of a defect in the bandgap at an energy, $E_{t}$, and with capture constants, $c_{n}$ and $c_{p}$, for electrons and holes, respectively, is governed by the rate equation (5),

$$
\begin{aligned}
\frac{d n_{t}}{d t}= & c_{n} n\left(N_{t}-n_{t}\right)-c_{n} N_{C} \exp \left[-\left(E_{c}-E_{t}\right) / k T\right] n_{t} \\
& -c_{p} p n_{t}+c_{p} N_{V} \exp \left[-\left(E_{t}-E_{v}\right) / k T\right]\left(N_{t}-n_{t}\right),
\end{aligned}
$$

where $N_{t}$ denotes the defect density, $n_{t}$ is the defect density occupied with an electron, and $n$ and $p$ are the free carrier densities. When performing a small signal analysis on Eq. (5), the carrier densities are decomposed in a steady state (designated with ' $=$ ') and an ac (designated with ' $\sim$ ') component,

$$
\begin{aligned}
n & =n^{=}+\tilde{n} e^{j \omega t} ; \quad p=p^{=}+\tilde{p} e^{j \omega t} ; \quad n_{t}=n_{t}^{=}+\tilde{n}_{t} e^{j \omega t} ; \\
\frac{d n_{t}}{d t} & =j \omega \tilde{n}_{t} e^{j \omega t} .
\end{aligned}
$$

In this way, Eq. (5) can be rewritten as Eq. (7),

$$
\begin{aligned}
j \omega \tilde{n}_{t} e^{j \omega t}= & c_{n}\left(N_{t}-n_{t}^{=}\right) n^{=}-c_{n} N_{C} \exp \left[-\left(E_{C}-E_{t}\right) / k T\right] n_{t}^{=} \\
& -c_{p} p^{=} n_{t}^{=}+c_{p} N_{V} \exp \left[-\left(E_{t}-E_{V}\right) / k T\right]\left(N_{t}-n_{t}^{=}\right) \\
& +\left\{c_{n}\left(N_{t}-n_{t}^{=}\right) \tilde{n}-c_{n} n^{=} \tilde{n}_{t}\right. \\
& -c_{n} N_{C} \exp \left[-\left(E_{C}-E_{t}\right) / k T\right] \tilde{n}_{t}-c_{p} \tilde{p} n_{t}^{=}-c_{p} p^{=} \tilde{n}_{t} \\
& \left.-c_{p} N_{V} \exp \left[-\left(E_{t}-E_{V}\right) / k T\right] \tilde{n}_{t}\right\} e^{j \omega t},
\end{aligned}
$$

neglecting the second and higher order terms in the small signal analysis. Since this equation should be valid for all values of $\omega$, it falls apart in a steady-state part which allows us to calculate the steady state occupation probability of the defect (8) and a small signal part which allows us to calculate the ac component of the defect occupation density (9),

$$
\begin{gathered}
f_{t}^{=}=\frac{n_{t}^{=}}{N_{t}}=\frac{c_{n} n^{=}+c_{p} \exp \left[-\left(E_{t}-E_{F p}\right) / k T\right] p^{=}}{\omega_{0}}, \\
\tilde{n}_{t}=\frac{c_{n}\left(N_{t}-n_{t}^{=}\right) \tilde{n}-c_{p} n_{t}^{=} \tilde{p}}{\omega_{0}+j \omega}
\end{gathered}
$$

where $\omega_{0}$ is given by

$$
\begin{aligned}
\omega_{0}= & c_{n} n^{=}\left(1+\exp \left[-\left(E_{F n}-E_{t}\right) / k T\right]\right) \\
& +c_{p} p^{=}\left(1+\exp \left[-\left(E_{t}-E_{F p}\right) / k T\right]\right) .
\end{aligned}
$$

It is easily checked that $f_{t}$ equals the Fermi function at the defect level when no bias is applied $\left(E_{F n}=E_{F p}\right)$, as was assumed in Ref. 4. 
The charging and discharging of a defect leads to a contribution to the current which can be expressed as Eq. (11) and which resembles Eq. (5),

$$
\begin{aligned}
J / q= & c_{n} n\left(N_{t}-n_{t}\right)-c_{n} N_{c} \exp \left[-\left(E_{c}-E_{t}\right) / k T\right] n_{t} \\
& +c_{p} p n_{t}-c_{p} N_{v} \exp \left[-\left(E_{t}-E_{v}\right) / k T\right]\left(N_{t}-n_{t}\right) .
\end{aligned}
$$

The same small signal approach used previously can be applied to Eq. (11). The capacitance due to the charging and discharging of the defects is then given by the imaginary part of the small signal current divided by the external ac voltage, $\tilde{u}_{e x t}$ and the angular frequency. The ac components of the free carrier densities, $\tilde{n}$ and $\tilde{p}$, are assumed to be in phase with the external applied signal (see Eq. (14)), and thus possess no imaginary part. Hence, the capacitance contribution due to charging and discharging of a defect state can be written as,

$$
\begin{aligned}
C^{\prime} & =\frac{\operatorname{Im}(\tilde{J})}{\omega \tilde{u}_{e x t}}=\frac{q \operatorname{Im}\left(\omega_{1} \tilde{n}_{t}\right)}{\omega \tilde{u}_{e x t}} \\
& =\frac{q N_{t}}{\tilde{u}_{e x t}} \frac{\omega_{1}}{\omega_{0}^{2}+\omega^{2}}\left[f_{t} c_{p} \tilde{p}-\left(1-f_{t}\right) c_{n} \tilde{n}\right],
\end{aligned}
$$

with $\omega_{1}$ given by

$$
\begin{aligned}
\omega_{1}= & -c_{n} n^{=}\left(1+\exp \left[-\left(E_{F n}-E_{t}\right) / k T\right]\right) \\
& +c_{p} p^{=}\left(1+\exp \left[-\left(E_{t}-E_{F p}\right) / k T\right]\right) .
\end{aligned}
$$

Equation (12) gives the contribution to the capacitance of a defect level at an energy, $E_{t}$, in the bandgap when the free electron and hole density are known. In order to calculate the total contribution of an energy distribution of defects, $N_{t}\left(E_{t}\right)$ this relation still has to be integrated along with energy and position. The frequency dependence of Eq. (12) shows that a defect can contribute to the capacitance if the angular frequency is low enough $\left(\omega \ll \omega_{0}\right)$ so that the charging and discharging of the defect can follow the applied ac signal. If the angular frequency is too high $\left(\omega \gg \omega_{0}\right)$, the defect will no longer contribute to the capacitance. Hence, we will assume the angular frequency to be low enough so that the defect contributes to the capacitance.

The ac components of the free carrier densities can be written as,

$$
\tilde{p}=p=\frac{q \tilde{u}_{p}}{k T} ; \quad \tilde{n}=n^{=} \frac{q \tilde{u}_{n}}{k T},
$$

with $q \tilde{u}_{n, p}$ representing the local shift of the quasi-Fermi levels with respect to the band edges when applying an additional ac signal to the junction. The contribution to the capacitance (12) can then be rewritten as,

$$
C^{\prime}=\frac{q^{2}}{k T} N_{t} \frac{\omega_{1}}{\omega_{0}^{2}}\left[f_{t} c_{p} p=\frac{\tilde{u}_{p}}{\tilde{u}_{e x t}}-\left(1-f_{t}\right) c_{n} n=\frac{\tilde{u}_{n}}{\tilde{u}_{e x t}}\right] .
$$

In order to facilitate the assessment of $C^{\prime}$ two variables are introduced,

$$
\gamma=\frac{c_{n} n^{=}}{c_{p} p^{=}} ; \quad U=E_{F n}-E_{F p} .
$$

The former reflects which charge carrier is dominant (weighed with the capture constants), the latter gives the local Fermi level splitting. In this way, $C^{\prime}$ can be rewritten as,

$$
C^{\prime}=\frac{q^{2}}{k T} \frac{N_{t}}{\tilde{u}_{e x t}}\left(W_{p} \tilde{u}_{p}+W_{n} \tilde{u}_{n}\right)
$$

where $W_{p}$ and $W_{n}$ are dimensionless parameters reflecting the contribution originating in the ac shifts in the hole and electron Fermi level, and which are only dependent on $\gamma, U$, and $E_{t}-E_{F p}$,

$$
\begin{aligned}
& W_{p}=\left(c_{p} p^{=}\right)^{2} \frac{\omega_{1}}{\omega_{0}^{3}}\left(\gamma+e^{-\left[\left(E_{t}-E_{F p}\right) / k T\right]}\right), \\
& W_{n}=-\left(c_{p} p^{=}\right)^{2} \frac{\omega_{1}}{\omega_{0}^{3}} \gamma\left(1+\gamma e^{\left[\left(E_{t}-E_{F p}-U\right) / k T\right]}\right) .
\end{aligned}
$$

When no dc bias voltage is applied, Walter et al. have shown that $W_{p}$ samples the defect distribution around the Fermi level, which leads to the conclusion that a defect state only contributes to the capacitance at the point where the defect energy level intersects the Fermi level. When Fermi level splitting is present, $W_{p}$ and $W_{n}$ still have a sampling character, however, the situation becomes more complicated. After some calculation, it can be seen that substituting $\gamma \rightarrow 1 / \gamma$ and $\left(E_{t}-E_{F p}\right)$ $\rightarrow\left(E_{F n}-E_{t}\right), \omega_{1} / \omega_{0}^{3}$ is transformed into $-\gamma^{2} \omega_{1} / \omega_{0}^{3}$ and thus, that $W_{n}$ is transformed into $W_{p}$ and vice versa. Hence, all statements which hold for $W_{p}$ also hold for $W_{n}$ when inverting $\gamma$ and interchanging $E_{F n}-E_{t}$ and $E_{t}-E_{F p}$.

In Figs. 1 and 2, $W_{p}$ is displayed as a function of $\gamma$ and $E_{F p}$ for both positive and negative Fermi level splitting. It can be seen that $W_{p}$ samples the defect distribution $N_{t}\left(E_{t}\right)$ around $E_{F p}$. Independent of the Fermi level splitting, there is only a contribution of $\tilde{u}_{p}$ to $C^{\prime}$ when $\gamma<1$. Under reverse bias voltage conditions the Fermi level splitting becomes negative $\left(\mathrm{U}<0\right.$; see Fig. 1) and $W_{p}$ disappears for $\gamma>\exp (U / k T)$. Under forward bias voltage conditions the Fermi level splitting is positive $(U>0$; Fig. 2). Under these conditions and when $\gamma \approx 1$, which corresponds to $p \approx n$ when $c_{n} \approx c_{p}$, the sampling is no longer limited to $E_{F p}$, but is extended to the

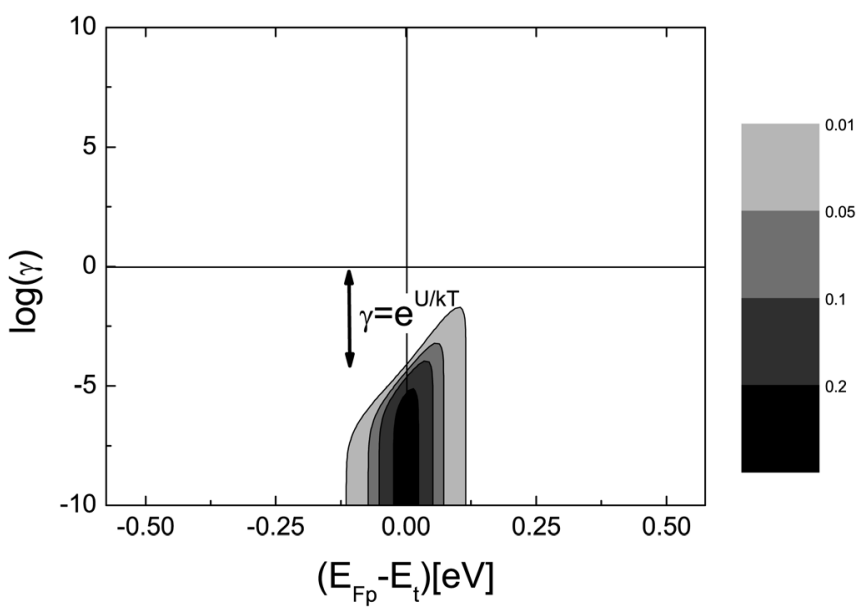

FIG. 1. Contribution to the capacitance, $W_{p}$, of the defect density due to ac shifts in the Fermi level for holes under reverse bias conditions: $U=-0.25 \mathrm{~V}, \gamma=c_{n} n^{=} / c_{p} p^{=}$; see Eq. (16). 


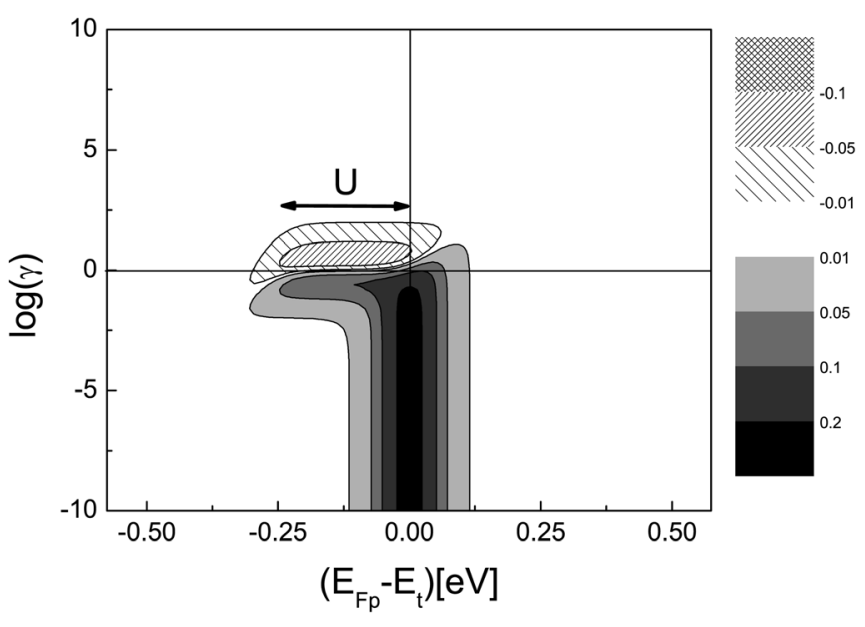

FIG. 2. Contribution to the capacitance, $W_{p}$, of the defect density due to ac shifts in the Fermi level for holes under forward bias conditions: $U=+0.25 \mathrm{~V}, \gamma=c_{n} n^{=} / c_{p} p^{=}$; see Eq. (16).

entire energy range of the width $|U|$ between $E_{F p}$ and $E_{F n}$. Fortunately this deviant sampling character only occurs for a limited $\gamma$-range. These conditions under forward bias are only present in a minor part of the junction.

In general, the statement that a defect only contributes to the capacitance at the intersection of the defect energy level with the (quasi-) Fermi level remains valid under nonequilibrium conditions. However, at positive Fermi level splitting an extra contribution can occur when the free electron and hole density are almost equal and at negative Fermi level splitting there is only a contribution to the capacitance if the absolute value of $\gamma$ is large enough.

To calculate the capacitance due to a band of defects distributed in energy, the expression for $C^{\prime}$ has to be integrated over the energies of the defect band. Under $p$-dominated circumstances, $W_{p}$ decreases exponentially with increasing distance of $E_{t}$ from $E_{F p}$, due to its sampling character. Hence, the integration limits of $E_{t}$ can be extended to $\pm \infty$. Additionally, assuming the defect distribution, $N_{t}\left(E_{t}\right)$ to be constant around the Fermi level, this leads to

$$
\begin{aligned}
C^{\prime \prime} & =\frac{q^{2}}{k T}\left[N_{t}\left(E_{F p}\right) \frac{\tilde{u}_{p}}{\tilde{u}_{e x t}} \int_{-\infty}^{+\infty} W_{p} d E_{t}+N_{t}\left(E_{F n}\right) \frac{\tilde{u}_{n}}{\tilde{u}_{e x t}} \int_{-\infty}^{+\infty} W_{n} d E_{t}\right] \\
& =q^{2}\left[N_{t}\left(E_{F p}\right) I_{p} \frac{\tilde{u}_{p}}{\tilde{u}_{e x t}}+N_{t}\left(E_{F n}\right) I_{n} \frac{\tilde{u}_{n}}{\tilde{u}_{e x t}}\right]
\end{aligned}
$$

where a short notation, $I_{p}$ and $I_{n}$, is introduced for the integrals over energy. Taking into account the relation between $W_{p}$ and $W_{n}$, it is easily shown that $I_{p}(\gamma)=I_{n}(1 / \gamma)$. When $\gamma<1$ (i.e., when $c_{n} n^{=}<c_{p} p^{=}$), $I_{n}$ disappears and only the term in $\tilde{u}_{p}$ remains.

In order to calculate $C^{\prime \prime}, N_{t}\left(E_{t}\right)$ was assumed to be constant around the Fermi level. For the sufficient dominance of one of the free charge carriers this can be quantified in a similar way as in Ref. 4 as being constant in a $4 k T$-wide energy interval around the Fermi level.

When $U<0$ (reverse bias), the contribution from $\tilde{u}_{p}$ to the capacitance, $C^{\prime}$ disappears when $\gamma>\exp (U / k T)$, as is expected from Fig. 1. Hence, this is also the case for $C^{\prime \prime}$, as can be seen in Fig. 3, where $I_{p}$ is drawn as a function of $\gamma$ and the Fermi level splitting $U$. Hence, $I_{p}$ disappears when $\gamma \gg \exp (U / k T)$ and $I_{p}=1$ when $\gamma \ll \exp (U / k T)$, and $\gamma=\exp (U / k T)$ corresponds to $I_{p}=1 / 3$.

Under forward bias conditions $(U>0)$ the additional contribution of states in the energy interval between $E_{F n}$ and $E_{F p}$ for small values of $\gamma$ leads to an error in the calculation of $C^{\prime \prime}$, where the value of $I_{p}$ is overestimated. If $\gamma$ exactly equals one, there is no contribution to the capacitance at all under all bias conditions. This behavior was already predicted for zero bias and was explained in terms of efficient recombination. 4

In a final step to calculate the contribution of a defect distribution to the capacitance of the device, an integration in space has to be carried out. Similarly as in Ref. 4, the upper limit of this integration is chosen as the depletion region edge $(x=w)$, since outside the depletion region only the minority carrier density is modulated by the ac-signal. The lower limit $\left(x=x_{1}\right)$ is determined as the point where the defect no longer contributes to the capacitance. There can be two reasons for this. First, under negative bias, this can be the point where $\gamma \approx \exp (U / k T)$. More often, however, this point is determined by the fact that a defect can only contribute to the capacitance if the angular frequency is sufficiently low, as will be assumed here. From Fig. 3, it can be seen that $I_{p}$ can often be assumed to be constant and almost equal to one. Hence, $I_{p}$ can be placed in front of the integral. The band bending determines a relation between the position of the Fermi level for holes/electrons and the valence/conduction band, $x\left(E_{F}\right)$, which can be substituted in the integral together with Eq. (19),

$$
C=\int_{x 1}^{w} C^{\prime \prime} d x=q^{2} \sum_{m=p \text { or } n}\left(I_{m} \frac{\tilde{u}_{m}}{\tilde{u}_{e x t}} \int_{E_{\omega, m}}^{E_{F m \infty}} N_{t}(E) \frac{d x}{d E} d E\right),
$$

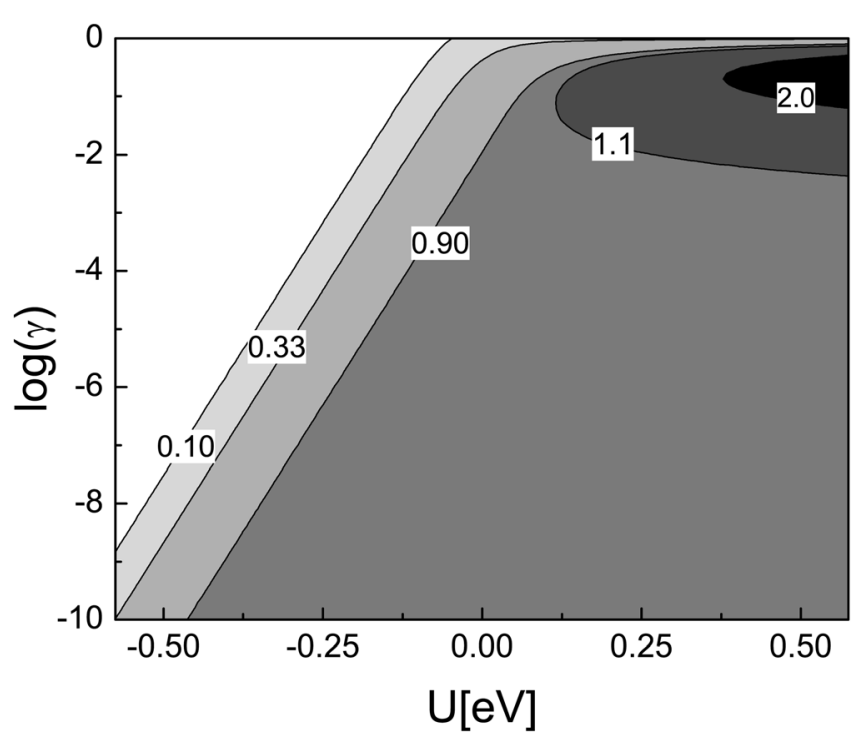

FIG. 3. Effectiveness, $I_{p}$, of the contribution of $\tilde{u}_{p}$ to the capacitance $C^{\prime \prime}$ as a function of $\gamma=c_{n} n=/ c_{p} p=$ and of the Fermi level splitting, $U$. 
where $m$ represents $n$ or $p, E_{F m \infty}$ represents the position of the Fermi level in the bulk of the $m$-type semiconductor, and $E_{\omega, m}$ is calculated from the inflection frequency $\omega_{0}$; see Eq. (10). Assuming one of the free carrier densities to be dominant and the corresponding quasi Fermi level to equal the defect energy level, the frequency at which a defect with this energy level no longer contributes to the capacitance can be calculated as,

$$
\begin{aligned}
\omega & =c_{p} p^{=}(1+1)=2 c_{p} N_{V} \exp \left[-\left(E_{F p}-E_{V}\right) / k T\right] \\
& =2 c_{p} N_{V} \exp \left[-E_{\omega, p} / k T\right], \\
\omega & =c_{n} n^{=}(1+1)=2 c_{n} N_{C} \exp \left[-\left(E_{C}-E_{F n}\right) / k T\right] \\
& =2 c_{n} N_{C} \exp \left[-E_{\omega, n} / k T\right] .
\end{aligned}
$$

Thus, $\mathrm{E}_{\omega, m}$ can then be calculated as

$$
\begin{aligned}
& E_{\omega, p}=E_{t}-E_{V}=k T \ln \frac{2 c_{p} N_{V}}{\omega}, \\
& E_{\omega, n}=E_{C}-E_{t}=k T \ln \frac{2 c_{n} N_{C}}{\omega} .
\end{aligned}
$$

The properties, $c_{p} N_{V}$ and $c_{n} N_{C}$, are called 'the attempt-toescape frequencies' and the one corresponding to the dominant charge carrier is often noted as $\nu_{0}$. Here, $\nu_{0}$ is proportional with $T^{2}$, and a generalized expression for $\mathrm{E}_{\omega}$ is given by

$$
E_{\omega}=k T \ln \left(\frac{\xi_{0} T^{2}}{\omega}\right)
$$

Both $E_{\omega}$ and $\xi_{0}$ can be found by making an Arrhenius diagram, drawing the inflection frequency multiplied by $T^{2}$ versus $1 / T$. The defect distribution can now be evaluated by taking the derivative with respect to the angular frequency, leading to

$$
\frac{d C}{d \omega}=-\frac{k T}{\omega} \frac{d C}{d E_{\omega, m}}=q^{2} \frac{k T}{\omega} \sum_{m=p \text { or } n}\left(I_{m} \frac{\tilde{u}_{m}}{\tilde{u}_{e x t}} N_{t}\left(E_{\omega, m}\right) \frac{d x}{d E_{\omega, m}}\right) .
$$

When the lower integration limit is determined by $\gamma$ $\approx \exp (U / k T)$ under negative bias, $d C / d \omega$ will vanish and it will not be possible to determine the defect distribution of this defect from the admittance spectroscopy under these circumstances. When the lower integration limit is determined by $E_{\omega, \xi}$, as assumed in Eqs. (20) and (24), it is possible to determine the defect distribution. Considering $\gamma<1$ (the analysis for $\gamma>1$ is similar), only the term in $\tilde{u}_{p}$ will remain and the defect distribution can be calculated as,

$$
N_{t}\left(E_{\omega}\right)=\frac{1}{q^{2}} \frac{\tilde{u}_{e x t}}{\tilde{u}_{p}} \frac{d E_{\omega}}{d x} \frac{1}{I_{p}} \frac{\omega}{k T} \frac{d C}{d \omega},
$$

omitting the second subscript of $E_{\omega}$ in order not to complicate the following expressions.

Assuming $\gamma$ is sufficiently small, $I_{p} \approx 1$ (Fig. 3), the expression can be elaborated further on, when making extra assumptions on the band bending. Three situations will be considered here: the 'constant-linear,' the 'linear,' and the 'parabolic' case.
The most straightforward assumption to make is $\tilde{u}_{p}=\tilde{u}_{\text {ext }}=$ constant in the entire depletion region, while considering linear band bending (as occurs in a nip junction). In this case (constant-linear), the position at which a defect is sensed with an energy, $E_{\omega}$, is determined by

$$
E_{\omega}=E_{F n \infty}-q V-q\left(V_{b i}-V\right) \frac{x}{w},
$$

with $V_{b i}$ as the built-in voltage, $V$ the externally applied dc bias voltage, and $w$ the depletion width. Here, $E_{F n \infty}$ is the position of the electron quasi Fermi level in the bulk of the $n$-type doped side of the junction. This leads to

$$
N_{t}\left(E_{\omega}\right)=-\frac{V_{b i}-V}{q w} \frac{d C}{d \omega} \frac{\omega}{k T} .
$$

In the linear case, again linear band bending (26) is assumed, however, now $\tilde{u}_{p}$ is assumed to vary linearly with $x$ as well,

$$
\frac{\tilde{u}_{p}}{\tilde{u}_{e x t}}=1-\frac{x}{w} .
$$

The linear profile of $\tilde{u}_{p}(x)=\widetilde{E_{F p}}(x)-\widetilde{E_{V}}(x)$ in Eq. (28) is made plausible as follows: At the $p$-side of the depletion region (at $x=w$ ), the hole quasi Fermi level, $E_{F p}$, is fixed with respect to the valence band and hence, $\widetilde{u_{p}}(w)=0$. Additionally, $E_{F p}(x)$ is supposed to be constant over the depletion layer, hence $\widetilde{E_{F p}}(x)=0$ for $0<x<w$. In, e.g., a nip junction, the electrostatic potential will drop linearly over the $i$-layer. Hence, it can be assumed that an applied small signal bias, $\tilde{u}_{\text {ext }}$ will cause a linear drop, $\widetilde{E_{V}}(x)$, over the depletion region. Hence, a linear behavior of $(x)$ is expected. Substituting (28) in (25) leads to

$$
N_{t}\left(E_{\omega}\right)=-\frac{\left(V_{b i}-V\right)^{2}}{w\left[q V_{b i}-E_{F n \infty}+E_{\omega}\right]} \frac{d C}{d \omega} \frac{\omega}{k T} .
$$

Similarly assuming parabolic bands and $\tilde{u}_{p}$ to vary parabolically with $x$, one obtains

$$
\begin{aligned}
E_{\omega} & =E_{F n \infty}-q V-2 q\left(V_{b i}-V\right) \frac{x}{w}+q\left(V_{b i}-V\right) \frac{x^{2}}{w^{2}}, \\
\frac{\tilde{u}_{p}}{\tilde{u}_{e x t}} & =\left(1-\frac{x}{w}\right)^{2} .
\end{aligned}
$$

Thus leading to

$$
N_{t}\left(E_{\omega}\right)=-\frac{2\left(V_{b i}-V\right)^{3 / 2}}{w \sqrt{q} \sqrt{q V_{b i}-E_{F n \infty}+E_{\omega}}} \frac{d C}{d \omega} \frac{\omega}{k T} .
$$

Making different assumptions on the band bending leads to different voltage dependencies of the derivative of the capacitance. The following combination of parameters, however, occurs in all band bending assumptions:

$$
-\frac{1}{w} \frac{d C}{d \omega} \frac{\omega}{k T}
$$

This will be called the 'scaled derivative' of the capacitance throughout the article. 


\section{Contribution of a (back contact) barrier}

Next to the presence of defects in the space-charge region, a back contact barrier (or any other barrier in the structure) can also introduce a capacitance step. ${ }^{8}$ Moreover, the presence of a non-ohmic back contact can explain several features commonly observed in a broad range of measurements of thin film solar cells. ${ }^{9}$ Hence, it is important to also investigate the voltage dependence of this process.

The capacitance signature of the influence of a back contact barrier, together with its characteristic frequency, $\omega_{0}$, using the same symbols as in Ref. 8 ( $G$ represents the conductance, the subscript $j$ represents junction, and $c$ is the contact), is given by

$$
\begin{aligned}
& C_{t}=\frac{C_{c} G_{j}^{2}+C_{j} G_{c}^{2}+\omega^{2} C_{j} C_{c}\left(C_{j}+C_{c}\right)}{\left(G_{j}+G_{c}\right)^{2}+\omega^{2}\left(C_{j}+C_{c}\right)^{2}}, \\
& \omega_{0}=\frac{1}{\tau_{c}}=\frac{G_{j}+G_{c}}{C_{j}+C_{c}} .
\end{aligned}
$$

Evaluating the admittance spectrum $-\omega \cdot d C_{t} / d \omega$ at $\omega_{0}$ renders

$$
-\left.\omega \frac{d C_{t}}{d \omega}\right|_{\omega=\omega_{0}}=\frac{1}{2} \frac{\left(G_{c} C_{j}-G_{j} C_{c}\right)^{2}}{\left(G_{c}+G_{j}\right)^{2}\left(C_{c}+C_{j}\right)} .
$$

All of the variables present in (33) and (34) are voltage dependent. Under reverse and moderate forward bias conditions, $G_{c}$ and $C_{c}$ can, however, be assumed to be voltage independent. Assuming the junction capacitance, $C_{j}$, to be a pure depletion capacitance, its value is proportional to $\left(V_{b i}-V\right)^{-1 / 2}$. Here, $G_{j}$ is proportional to $\exp \left(q V_{j} / n k T\right)$, with $n$ representing the ideality factor and $V_{j}$ the voltage drop over the junction. Assuming the back contact to not block the current $\left(G_{c} \gg G_{j}\right)$, the characteristic frequency is given by

$$
\omega_{0}=\frac{G_{c}}{C_{j}+C_{c}} .
$$

The derivative of the capacitance at the characteristic frequency is then given by

$$
-\left.\omega \frac{d C_{t}}{d \omega}\right|_{\omega=\omega_{0}}=\frac{1}{2} \frac{C_{j}^{2}}{C_{j}+C_{c}} .
$$

If $C_{j}<C_{c}$, the characteristic frequency does not depend on the external applied voltage. The derivative of the capacitance is then proportional to $w^{-2}$, and thus to $\left(V_{b i}-V\right)^{-1}$. If $C_{j}>C_{c}$, the characteristic frequency is proportional to $\left(V_{b i}-V\right)^{1 / 2}$. The derivative of the capacitance is then proportional to $w^{-1}$ and thus to $\left(V_{b i}-V\right)^{-1 / 2}$.

The characteristic frequency is temperature dependent. The current across a Schottky barrier can be described by, ${ }^{15}$

$$
J \approx q N_{V} \mu_{h} E \exp \left(-q \Phi_{B} / k T\right)\left[\exp \left(-q V_{C} / k T\right)-1\right],
$$

with $E$ representing the electric field strength and $\Phi_{B}$ the barrier height. Here, $V_{C}$ represents the voltage drop over the back contact barrier: $V=V_{C}+V_{j}$. As shown by Niemegeers and Burgelman, ${ }^{16} V_{C}=0$ for reverse and moderate forward dc voltages, and linearly increases for higher forward voltages. As can be seen in (37), $G_{c}$ and the characteristic frequency are thermally activated and has an activation energy, $E_{\omega}$,

$$
E_{\omega}=q\left(\Phi_{B}+V_{C}\right)=k T \ln \left(\frac{\xi_{0} T^{m}}{\omega_{0}}\right)
$$

where the exponent, $m$, depends on the temperature dependence of the mobility, $\mu_{h}$, and the effective density of states, $N_{V}$. Assuming the mobility to be temperature independent, $m$ equals $3 / 2$. The characteristic frequency, $\omega_{c}$, has the same activation energy because $C_{j}$ and $C_{c}$ are not thermally activated; see Eq. (35).

\section{Overview of the voltage dependencies}

There are different processes which can give a step in the capacitance as a function of frequency. The derivative of the capacitance has a specific voltage dependence as can be seen from Eqs. (3), (27), (29), (31), and (36). These voltage dependencies are summarized in Table I. Often the voltage dependence is linked with the depletion width. For a $n^{+} p$-junction, in a first approach, this depletion width can be calculated as,

$$
w=\left(\frac{2 \varepsilon}{q N_{A}}\left(V_{b i}-V\right)\right)^{1 / 2},
$$

with $N_{A}$ representing the shallow acceptor doping density. However, since the depletion width is not always determined by the shallow doping density alone, it is often better to determine $w$ immediately from the capacitance measurement at a well-chosen frequency. Therefore, the proportionality to $w$ is once explicitly mentioned and once replaced by (39) in Table I. The relations listed for the back contact are valid for reverse and moderate forward voltages. At high forward voltage the main voltage drop will occur over the barrier instead of the junction and the role of the junction and barrier can be interchanged.

\section{Spatial defect density variations}

Equation (25) expresses a relation between the defect density at the intersection of the defect level energy and the Fermi level. The spatial position of this intersection point varies with the defect energy level. It is thus not straightforward to interpret the derivative of the capacitance as a

TABLE I. Overview of voltage dependences of the derivative of the capacitance.

\begin{tabular}{lcc}
\hline \hline Effect & Assumption & 'Derivative': $\omega \frac{d C}{d \omega}$ \\
\hline Carrier relaxation & $t \gg w$ & $\propto w^{-1} \propto\left(V_{b i}-V\right)^{-1 / 2}$ \\
& $w \gg t$ & Voltage independent \\
Back contact/barrier & $C_{j} \gg C_{c}$ & $\propto w^{-1} \propto\left(V_{b i}-V\right)^{-1 / 2}$ \\
& $C_{c} \gg C_{j}$ & $\propto w^{-2} \propto\left(V_{b i}-V\right)^{-1}$ \\
Defect distribution & Constant-linear & $\propto \frac{w}{V_{b i}-V} \propto\left(V_{b i}-V\right)^{-1 / 2}$ \\
& Linear & $\propto \frac{w}{\left(V_{b i}-V\right)^{2}} \propto\left(V_{b i}-V\right)^{-3 / 2}$ \\
& Parabolic & $\propto \frac{w}{\left(V_{b i}-V\right)^{3 / 2}} \propto\left(V_{b i}-V\right)^{-1}$ \\
\hline \hline
\end{tabular}


measure for the defect density at a certain position, unless the defect density can be assumed to be spatially uniform. Varying the applied dc-voltage, however, shifts the intersection point of the Fermi level with the defect energy level, which allows us to assess the assumption of a uniform defect density.

In order to be able to calculate the voltage dependence of the derivative of the capacitance step, starting from Eq. (25), additional assumptions about the band bending have to be used, which lead to different results. Hence, fitting the voltage dependence of the derivative of the capacitance to the models of Eqs. (27), (29), and (31) and assessing the goodness of fit, can provide a clue about the band bending in the sample. Moreover, it gives an estimate for the parameters, $N_{t}$ and $V_{b i}$. $E_{F n \infty}$ is not a true fitting parameter, but could be calculated from (an estimation) of the shallow doping density at the $n$-side of the junction. Fitting these parameters rather than estimating them improves the accuracy of the final result. ${ }^{17}$ Since the fitting requires a spatially uniform value of $N_{t}$ it should be performed at an energy, $E_{\omega}$, where this can be assumed. This energy might be different from the energy at which the derivative of the capacitance reaches a maximum. Using the parameters from the fitting procedure together with Eqs. (26)-(31), an estimate of the spatial variation of the defect density belonging to a specific defect energy level, $E_{\omega}$, can be made. ${ }^{18}$ However, care should be taken with the assumptions made.

An alternative way ${ }^{19}$ to obtain to estimates of $N_{t}, V_{b i}$, and defect distribution profiles is through capacitancevoltage measurements $(C-V)$ and through drive level capacitance profiling (DLCP). In its most straightforward interpretation, $C-V$ profiling leads to the determination of the shallow doping density and the built-in voltage. ${ }^{15}$ As was demonstrated by Kimerling ${ }^{20}$ the presence of deep defects has an influence on these profiles. Hence, $C-V$ profiling can be used to determine the defect density and also the built-in voltage. Due to large non-uniformities in the apparent doping densities calculated from $C-V$ measurements on chalcopyrite solar cells it is, however, not always possible to determine the built-in voltage with reasonable accuracy. A straightforward application of the method proposed by Kimerling for a spatial uniform defect density allows us to calculate the shallow doping density, but is unable to detect the defect density close to the junction.

Compared to $C-V$ profiling, the determination of the defect density from the derivative of the capacitance to the frequency is hampered by the need for an assumption on the band bending. The described fitting procedure can be performed at any value of $E_{\omega}$, which allows us to select an energy where the defect density can be considered more or less spatially uniform. The spectra of the derivative of the capacitance allows us to see the contribution of different defects with different defect energy levels to the total defect density. This is not so easily accessible with $C-V$ profiling where the link between the defect level energy and density (in $\mathrm{cm}^{-3} / \mathrm{eV}$ ) is not so straightforward. It is possible to calculate the energy distribution of a defect at a certain position, starting from the results of DLCP measurements under different frequency/temperature conditions. ${ }^{3}$

\section{Issues when determining the admittance spectrum}

When measuring capacitance as a function of bias voltage and frequency, several issues which influence the measurement result have to be taken into account and possibly corrected.

Whenever a significant dc current flows through the junction, the low frequency phase angle of the admittance vector is close to zero. ${ }^{21}$ The imaginary part of the ac current phasor is then much smaller than the real part which complicates the extraction of the capacitance from the admittance. Under zero or reverse bias, this effect only occurs at frequencies well below the frequency window which is used in admittance spectroscopy. Under forward bias conditions or under strong illumination conditions, however, this can render the measurements at moderate and low frequencies unreliable. A similar effect occurs in the numerical simulation, where the effect is caused by numerical inaccuracies.

In the high frequency regime, parasitic network elements cause a resonance in the admittance which destroys the signature of the capacitance-frequency profile. ${ }^{22}$ Unless difficulties in determining the capacitance at lower frequencies arise, we can compensate for these effects. ${ }^{23}$

Considering Table I, it is important to obtain a good estimate of the depletion width, $w$, especially since $w$ is bias dependent $\left(w \propto \sqrt{V_{b i}-V}\right)$. The depletion width can be determined from the capacitance,

$$
w=\frac{\varepsilon}{C} .
$$

Equation (40) assumes that the depletion layer is the only contribution to the capacitance, hence this capacitance should be measured at a sufficiently high frequency to avoid the contributions of defects, but low enough to avoid the contribution of parasitic elements.

Under forward bias conditions the determination of the defect density from admittance spectroscopy suffers from additional complications. In all of the previous equations it was assumed that the applied dc voltage entirely drops over the junction of the cell. Under forward bias, a substantial part of the voltage, however, can drop over the series resistance(s) present in the cell and the measurement setup. Moreover, the diffusion capacitance can also contribute.

\section{Numerical simulations}

The relations listed in Table I are tested with numerical simulations using the solar cell device simulator, SCAPS ${ }^{11}$ (version 2.9). ${ }^{12}$ The structure which is used is representative for a $\mathrm{Cu}(\mathrm{In}, \mathrm{Ga}) \mathrm{Se}_{2}$-based thin film solar cell (based on a simplified version of the structure presented in Ref. 24), and an overview of the main parameters of the absorber and buffer layer is given in Table II. Four situations are investigated. In the first two scenarios, a spatially uniform acceptor defect with a defect energy of $0.35 \mathrm{eV}$ above the valence band is introduced in the absorber layer with a defect density of $10^{14} \mathrm{~cm}^{-3}$ ('Low Nt') and $10^{16} \mathrm{~cm}^{-3}$ ('High $\mathrm{Nt}^{\prime}$ ). The capture cross section, $\sigma_{p}=\sigma_{n}$, of this defect is $10^{-15} \mathrm{~cm}^{2}$. In a third scenario, the same defect is introduced with a non-uniform defect 
TABLE II. Overview of the main parameters used in the device simulation. The structure is based on a simplified version of the structure presented in Ref. 24 and consists of an absorber, buffer, and window layer.

\begin{tabular}{lcc}
\hline \hline & CIGS-absorber & CdS-buffer \\
\hline Thickness $(\mu \mathrm{m})$ & 2.3 & 0.1 \\
Band gap $(\mathrm{eV})$ & 1.1 & 2.42 \\
$N_{C}, N_{V}\left(\mathrm{~cm}^{-3}\right)$ & $10^{19}$ & $10^{19}$ \\
$v_{t h}\left(\mathrm{~cm}^{-3}\right)$ & $10^{7}$ & $10^{7}$ \\
$\mu_{n}\left(\mathrm{~cm}^{2} / \mathrm{V} / \mathrm{s}\right)$ & 100 & 100 \\
$\mu_{p}\left(\mathrm{~cm}^{2} / \mathrm{V} / \mathrm{s}\right)$ & 25 & 25 \\
Shallow doping density $\left(\mathrm{cm}^{-3}\right)$ & $10^{15}$ (acceptors) & $3 \times 10^{17}$ (donors) \\
\hline
\end{tabular}

density ('NonUniform'), decreasing exponentially with a characteristic length of $0.1 \mu \mathrm{m}$ from $10^{16} \mathrm{~cm}^{-3}$ at the bufferabsorber interface to $10^{10} \mathrm{~cm}^{-3}$ and remaining constant onwards. In a last scenario, there is no contribution of any defect to the capacitance (neutral defects), but a back contact barrier of $0.3 \mathrm{eV}$ relative to the Fermi level is introduced ('Back Barrier').

Capacitance-frequency simulations at various temperatures under thermal equilibrium have been performed on these structures. The Arrhenius diagrams are shown in Fig. 4. From the slope and intercept, the activation energies and attempt to escape frequencies (at $300 \mathrm{~K}$ ) are deduced; see Table III. In the case of the defect being responsible for the capacitance step, the attempt to escape frequency agrees with the parameters set $\left(\sigma_{p} v_{t h} N_{V}\right)$. The activation energy is underestimated in the 'high $\mathrm{Nt}^{\prime}$ ' case; this is a result of the dispersion of the derivative of the capacitance step at high defect densities. The activation energy in the 'Back Barrier' case is also slightly underestimated.

Capacitance-frequency simulations at various dc bias voltages are performed on all structures. The maximum of the scaled derivative of the capacitance (32) is tracked as a function of the voltage, as shown in Fig. 5. These values are fit to the voltage dependencies listed in Table I. In the case of a spatially uniform defect distribution being responsible for the capacitance step ('High $\mathrm{Nt}$ ' and 'Low Nt') the best fit

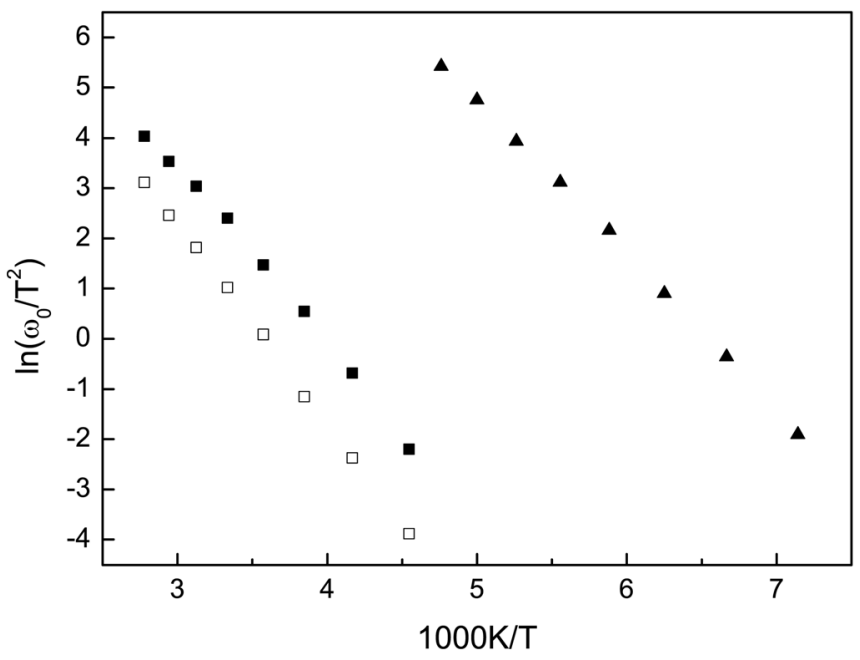

FIG. 4. Arrhenius diagrams deduced from the capacitance-frequency simulations of the structures 'High Nt' (solid squares), 'Low Nt' (empty squares), and 'Back Barrier' (triangles).
TABLE III. Parameters extracted from the Arrhenius diagram of the simulation results (activation energy, $E_{A}$, intercept with the ordinate, $\xi_{0}$, and attempt to escape frequency at $\left.300 \mathrm{~K}, \nu_{0}\right)$.

\begin{tabular}{lccc}
\hline \hline & $E_{A}(\mathrm{eV})$ & $\xi_{0}\left(\mathrm{~Hz} / \mathrm{K}^{2}\right)$ & $\nu_{0}(\mathrm{~Hz})$ at $300 \mathrm{~K}$ \\
\hline High Nt & 0.30 & $1.20 \times 10^{6}$ & $5.04 \times 10^{10}$ \\
Low Nt & 0.34 & $1.48 \times 10^{6}$ & $6.65 \times 10^{10}$ \\
Back barrier & 0.26 & $1.04 \times 10^{9}$ & \\
\hline \hline
\end{tabular}

is obtained using Eq. (31). Using Eq. (27) a reasonable, but worse fit is found, and using Eq. (29) no good fit was obtained. This should be the case since the band bending in the simulated structures was indeed parabolic. In the case of a non-uniform defect density, 'NonUniform,' no good fit could be made with any of the models. For the case of a back contact barrier, 'Back Barrier,' the best fit was obtained assuming $C_{c} \gg C_{j}$. An overview of the fitted parameters with $95 \%$ confidence intervals is given in Table IV. There is a good agreement of the built-in voltage determined by the fitting and the built-in voltage determined from the $C-V$ simulations.

Analyzing results from a single defect level, which represents a very narrow defect distribution, the assumption of $N_{t}$ being constant within an energy interval of $\pm 2 k T$ is violated, so the resulting spectrum yields a broad peak instead of a Dirac distribution. One can prove that the full width at half maximum of a single defect level will have a minimum value given by $F W H M$,

$$
F W H M=k T \ln \left(\frac{1+\sqrt{2}}{1-\sqrt{2}}\right) \approx 1.76 k T .
$$

Hence, the defect density expressed in $\mathrm{cm}^{-3} / \mathrm{eV}$ has no physical meaning for a single defect level, but one should integrate the area under the peak. The results of this integration are also shown in Table IV and agree well with the actual value of the defect density.

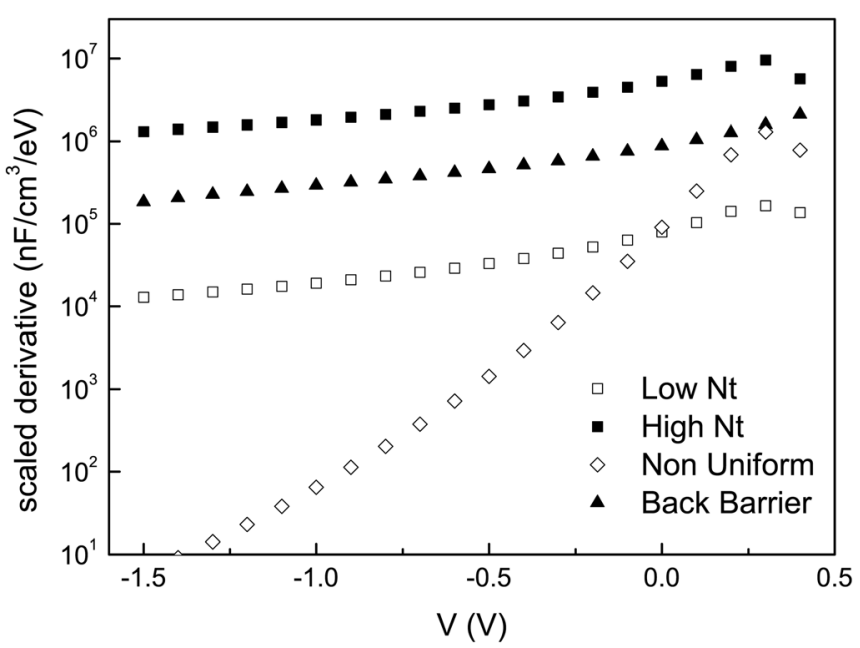

FIG. 5. Voltage dependence of the scaled derivative of the capacitance: Eq. (32). Simulations performed at $300 \mathrm{~K}$ for 'High Nt' (solid squares), 'Low $\mathrm{Nt}$ ' (empty squares), and 'NonUniform' (empty diamonds). Simulations performed at $200 \mathrm{~K}$ for 'Back Barrier' (triangles) in order to enhance the effect of a back contact barrier. 
TABLE IV. Parameters obtained from fitting the voltage dependence of the derivative of the capacitance to the models proposed with $95 \%$ confidence intervals (built-in voltage, $V_{b i}$, and uniform defect density, $N_{t}$ ) together with the built-in voltage determined from $C-V$ simulations $\left(V_{b i}{ }^{C V}\right)$.

\begin{tabular}{lcccc}
\hline \hline & $V_{b i}(\mathrm{~V})$ & $N_{t}\left(\mathrm{~cm}^{-3} / \mathrm{eV}\right)$ & $N_{t}\left(\mathrm{~cm}^{-3}\right)$ & $V_{b i}{ }^{C V}(\mathrm{~V})$ \\
\hline High Nt & $1.025 \pm 0.021$ & $1.17 \times 10^{17 \pm 0.007}$ & $1.04 \times 10^{16 \pm 0.007}$ & 1.124 \\
Low Nt & $0.633 \pm 0.004$ & $3.09 \times 10^{15 \pm 0.031}$ & $2.07 \times 10^{14 \pm 0.031}$ & 0.623 \\
Back barrier & $0.906 \pm 0.012$ & & & 1.035 \\
\hline \hline
\end{tabular}

Capacitance-voltage simulations have also been carried out. The apparent doping density profiles are shown in Fig. 6. For 'HighNt' the defect concentration can be determined as $9 \times 10^{15} \mathrm{~cm}^{-3}$ according to the method described by Kimerling. ${ }^{20}$ For 'LowNt' the defect density cannot be determined from this plot. The nonuniformity in the defect density of 'NonUniform' is slightly visible in the apparent doping density profile, but is not as obvious as in the voltage dependence of the derivative of the capacitance (Fig. 5).

\section{EXPERIMENT}

\section{Set-up}

The procedure developed above is applied to two different $\mathrm{Cu}(\mathrm{In}, \mathrm{Ga}) \mathrm{Se}_{2}$-based thin film solar cells prepared at EMPA with dissimilar buffer-absorber interfaces. Sample 1 was fabricated using a modified three stage co-evaporation process and has a $30 \mathrm{~nm} \mathrm{In}_{2} \mathrm{~S}_{3}$-buffer deposited by ultrasonic spray pyrolysis. ${ }^{25}$ Sample 2 has been fabricated according to the conventional three stage co-evaporation process used in EMPA with a $100 \mathrm{~nm}$ CdS buffer layer deposited in a chemical bath. ${ }^{26}$ These samples have been selected in order to provide results on samples with different buffer-absorber interfaces, hence, a complete description of the devices has not been provided.

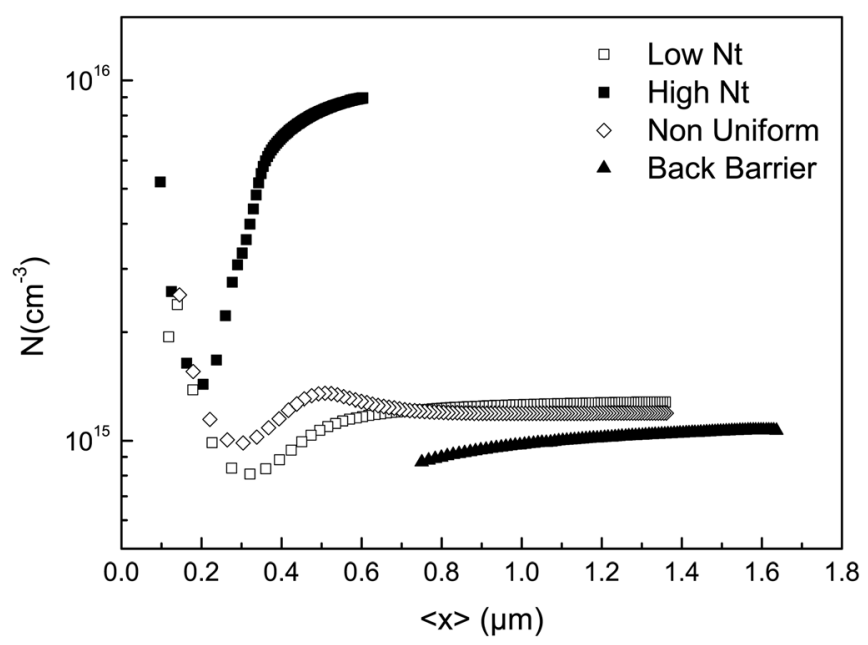

FIG. 6. Apparent doping density profiles calculated from capacitance voltage simulations $(f=1 \mathrm{MHz}, V=-1 \mathrm{~V} \rightarrow 0.6 \mathrm{~V}$ ) for 'High $\mathrm{Nt}$ ' (solid squares), 'Low Nt' (empty squares), 'NonUniform' (empty diamonds), and 'Back Barrier' (triangles). In the 'Back Barrier'-case convergence errors occurred when $V>0.54 \mathrm{~V}$.
Four point admittance measurements were performed using a Hewlett Packard 4192 A impedance analyzer. The admittance data were measured using a $30 \mathrm{mV}$ test signal and evaluated assuming a parallel equivalent circuit. The sample was mounted in a $\mathrm{N}_{2}$-coolable cryostat varying the temperature from 100 to $360 \mathrm{~K}$ in steps of $20 \mathrm{~K}$, and relaxed for one hour before cooling down. The frequency was varied from $100 \mathrm{~Hz}$ to $10 \mathrm{MHz}$. Afterwards, data points which are hampered by parasitic network elements, or which lead to a phase angle smaller than $10^{\circ}$ have been discarded. All measurements were performed under dark conditions and the applied dc voltage ranged from -2.0 to $0.8 \mathrm{~V}$. Before every measurement, an interval of at least $30 \mathrm{~s}$ was adopted in order to allow slow capacitance transients to extinguish.

\section{RESULTS AND DISCUSSION}

Capacitance-frequency relations under different dc bias conditions have been measured at various temperatures. Figure 7 shows the results for sample 1 under thermal equilibrium. For sample 1, a distinct capacitance step is visible for temperatures ranging from 160 to $220 \mathrm{~K}$ for all applied bias voltages. For sample 2, a similar step is only visible when a bias voltage of at least $0.3 \mathrm{~V}$ is applied. For both samples at higher temperatures $(\mathrm{T}>250 \mathrm{~K})$ the capacitance decreases slowly with increasing frequency.

The Arrhenius diagrams for the distinct step at low temperatures is shown in Fig. 8. An overview of the activation energies and attempt to escape frequencies is given in Table V. The attempt to escape frequency does not vary much with the applied voltage and will be assumed to be bias independent. The different $C$ - $f$ graphs are transformed to graphs of the scaled derivative (32) as a function of the $E_{\omega}$ using Eq. (23) with $\xi_{0}=1.1 \times 10^{6} \mathrm{~Hz} / \mathrm{K}^{2}$ for sample 1 and $\xi_{0}=3.1 \times 10^{4} \mathrm{~Hz} / \mathrm{K}^{2}$ for sample 2 . This approach is not entirely valid for Eqs. (4) and (38) since the temperature dependence of the mobility is unknown, but it can be adopted since the Arrhenius-diagrams are straight. For a given sample and applied bias voltage the curves belonging to different temperatures have a considerable overlap with each other.

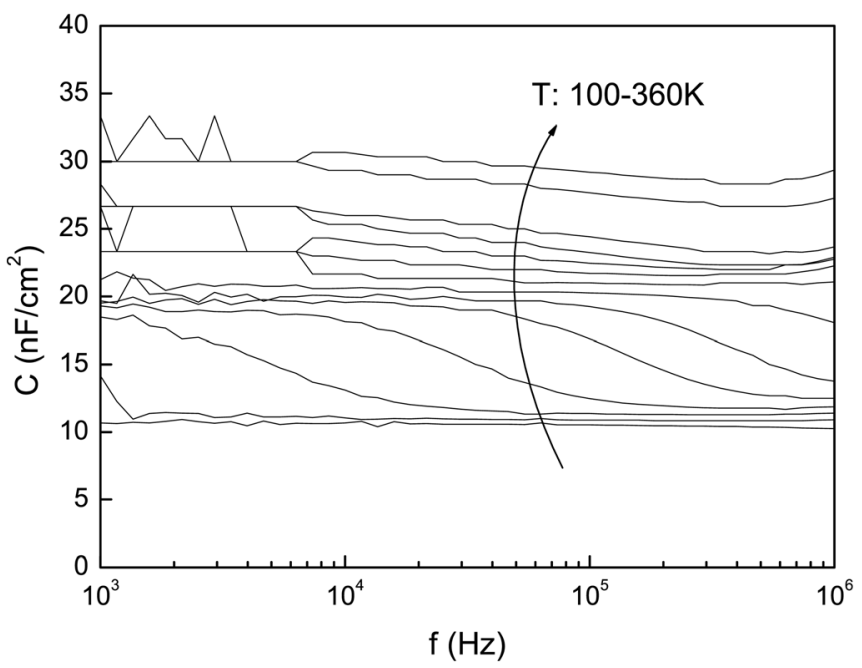

FIG. 7. Capacitance frequency relations measured at different temperatures on sample 1 under thermal equilibrium conditions. 


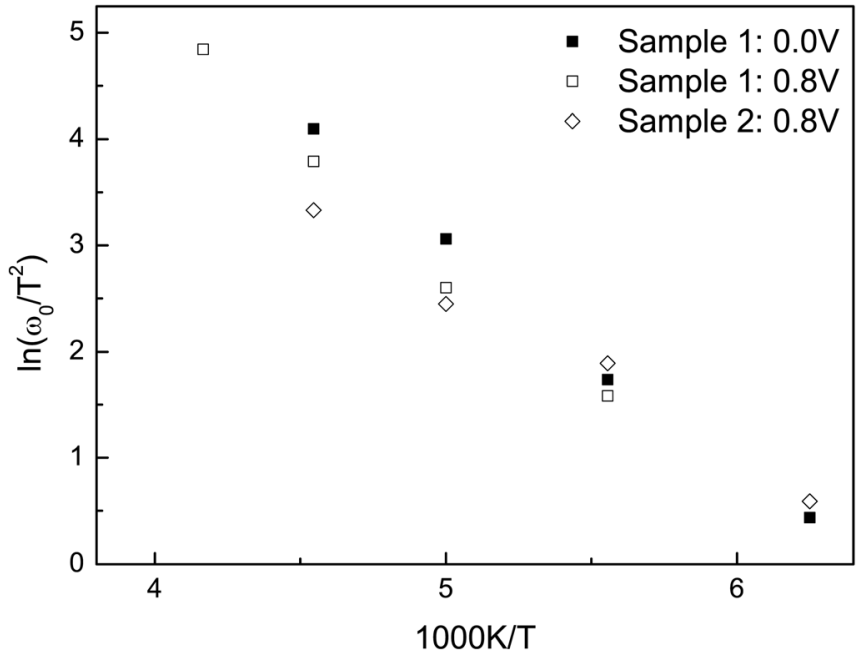

FIG. 8. Arrhenius diagrams for the capacitance step at low temperatures. The capacitance step is only visible at high enough forward voltages in sample 2 (the Arrhenius diagram at $0.8 \mathrm{~V}$ is shown) and is visible at all voltages in sample 1 (the Arrhenius diagram at 0.0 and $0.8 \mathrm{~V}$ is shown).

Combining them leads to a single curve for every voltage; an overview of these curves after removing the noise is shown in Fig. 9. The capacitance step at low temperatures leads to a peak in the scaled derivative versus $E_{\omega}$-curve. For sample 2, this peak is absent for reverse and moderate forward voltages. The activation energy belonging to this peak is slightly dependent on the applied bias voltage, however not significantly. The decrease of the capacitance with increasing frequency at higher temperatures leads to a broad band for $E_{\omega}>0.2 \mathrm{eV}$.

The value of the scaled derivative at the peak position as a function of applied bias voltage is tracked; see Fig. 10. For sample 2, the curves of the scaled derivative as a function of $E_{\omega}$ (Fig. 9) are more or less parallel at higher values of $E_{\omega}$, indicating the presence of defect with a broad energy distribution. The value of the scaled derivative at $0.32 \mathrm{eV}$ as a function of the voltage is also shown in Fig. 10. The voltage dependence of the scaled derivative at the peak position for sample 1 and at $0.32 \mathrm{eV}$ for sample 2 are fit to the relations listed in Table I (it is impossible to make a good fit at the peak position for sample 2). In both cases it is possible to obtain a reasonable fit for $V<0.25 \mathrm{~V}$ with the relations (27) and (31), but not with relation (29). An overview of the fitted parameters (assuming $E_{F n \infty}=1 \mathrm{eV}$ in Eq.(31)) is given in Table VI, together with the built-in voltage determined from capacitance voltage measurements at $200 \mathrm{~K}$. The fitted relations are also displayed in Fig. 10. Even though both

TABLE V. Parameters extracted from the Arrhenius diagram of measurement results (activation energy, $E_{A}$, intercept with the ordinate, $\xi_{0}$, and attempt to escape frequency at $300 \mathrm{~K}, \nu_{0}$ ).

\begin{tabular}{lccc}
\hline \hline & $E_{A}(\mathrm{eV})$ & $\xi_{0}\left(\mathrm{~Hz} / \mathrm{K}^{2}\right)$ & $\nu_{0}(\mathrm{~Hz})$ at 300 K \\
\hline Sample 1 (0 V) & 0.19 & $1.05 \times 10^{6}$ & $4.75 \times 10^{10}$ \\
Sample 1 (0.8 V) & 0.20 & $2.09 \times 10^{6}$ & $9.45 \times 10^{10}$ \\
Sample 2 (0.8 V) & 0.13 & $3.11 \times 10^{4}$ & $1.40 \times 10^{9}$ \\
\hline \hline
\end{tabular}

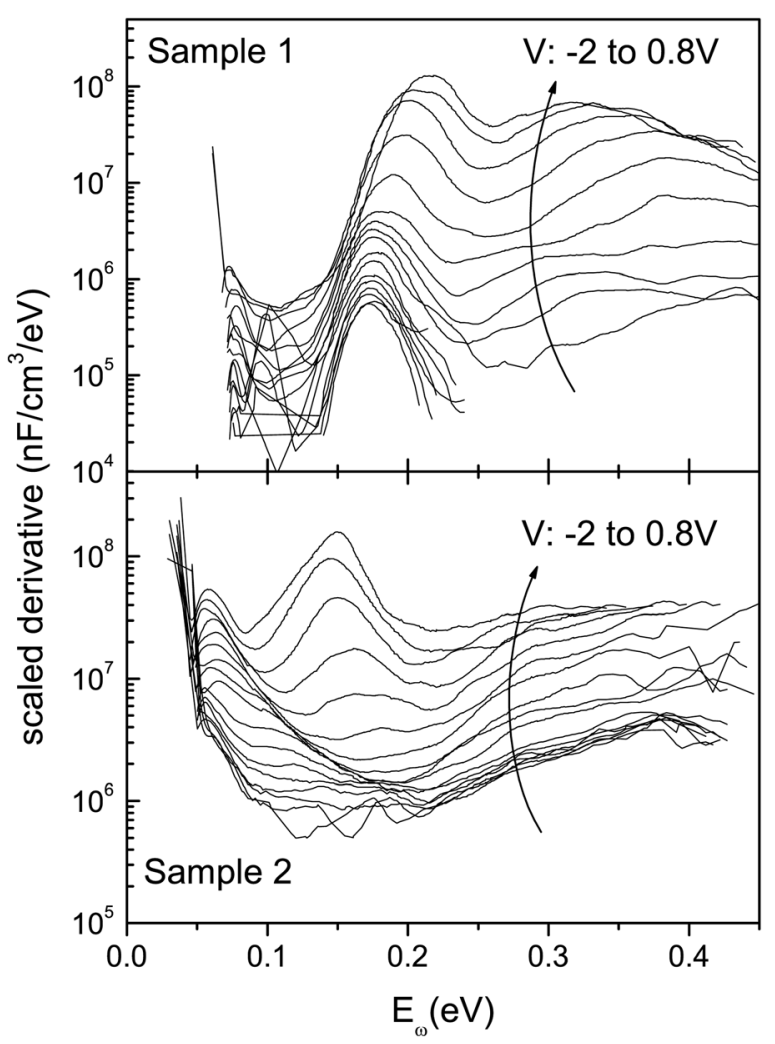

FIG. 9. Scaled derivative of the capacitance, Eq. (32), for both CIGS samples under various bias voltage conditions. Each graph is a composition of the graphs at different temperatures. The composite graphs have been merged and smoothed with a specially designed algorithm.

relations give a good result, the value for the built-in voltage determined from Eq. (31) is about $0.5 \mathrm{~V}$ higher than from Eq. (27), and the assumed defect density is estimated as eight times higher. The value of the built-in voltage determined from $C-V$ measurements agrees with the fitted value using

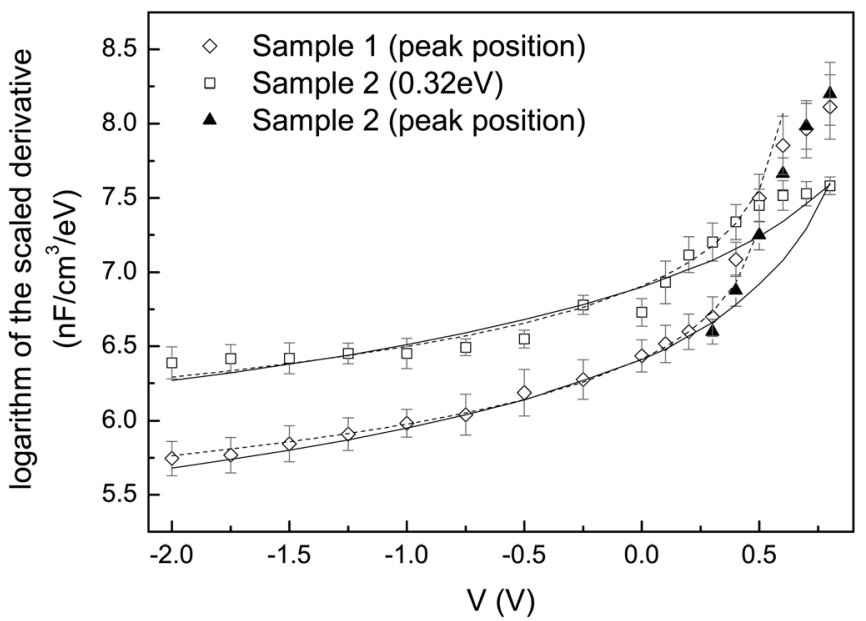

FIG. 10. Voltage dependence of the scaled derivative of the capacitance; see Eq. (32). For both samples the local maximum corresponding with the capacitance step at low temperatures is shown (peak position). Additionally, for sample 2, the scaled derivative corresponding to $E_{\omega}=0.32 \mathrm{eV}$ is shown. The results of the fitting to Eqs. (27) (dashed line) and (31) (solid line) is also shown. These fits implicitly also check the relationships for the back barrier case and the carrier relaxation case since the voltage dependence is similar. 
TABLE VI. Parameters obtained from fitting the voltage dependence of the derivative of the capacitance to the models proposed with $95 \%$ confidence intervals (built-in voltage, $V_{b i}$, and uniform defect density, $N_{t}$ ) together with the built-in voltage determined from $C-V$ measurements.

\begin{tabular}{lcl}
\hline \hline & \multicolumn{1}{c}{ Sample 1 } & \multicolumn{1}{c}{ Sample 2 } \\
\hline Fit to parabolic model (31) & & \\
$V_{b i}(\mathrm{~V})$ & $0.969 \pm 0.047$ & $1.219 \pm 0.430$ \\
$N_{t}\left(\mathrm{~cm}^{-3} / \mathrm{eV}\right)$ & $8.07 \times 10^{16 \pm 0.038}$ & $1.83 \times 10^{17 \pm 0.072}$ \\
$N_{t}\left(\mathrm{~cm}^{-3}\right)$ & $3.53 \times 10^{15 \pm 0.038}$ & $\ldots$ \\
Fit to linear-constant model $(27)$ & \\
$V_{\text {bi }}(\mathrm{V})$ & $0.576 \pm 0.029$ & $0.644 \pm 0.114$ \\
$N_{t}\left(\mathrm{~cm}^{-3} / \mathrm{eV}\right)$ & $9.30 \times 10^{15 \pm 0.028}$ & $3.203 \times 10^{16 \pm 0.087}$ \\
$N_{t}\left(\mathrm{~cm}^{-3}\right)$ & $4.07 \times 10^{14 \pm 0.038}$ & $\ldots$ \\
From Capacitance-Voltage & measurements & \\
$V_{b i}(\mathrm{~V})[\mathrm{T}: 200 \mathrm{~K}]$ & 0.971 & 1.391 \\
\hline \hline
\end{tabular}

Eq. (31), indicating that the parabolic model is more realistic in this case than the constant-linear model.

The apparent doping density profiles extracted from capacitance voltage measurements at $200 \mathrm{~K}$ are shown in Fig. 11. Applying the theory presented in Ref. 20 for sample 2, a shallow doping density of $2.5 \times 10^{16} \mathrm{~cm}^{-3}$, and a defect density of $5 \times 10^{16} \mathrm{~cm}^{-3}$ is found. For sample 1 , only the shallow doping density can be extracted as $3 \times 10^{15} \mathrm{~cm}^{-3}$. Integrating the calculated defect density under the peak in Fig. 9, a defect density of $3.53 \times 10^{15} \mathrm{~cm}^{-3}$ is found, assuming parabolic band bending, which agrees with the apparent doping density (Fig. 11).

Since the capacitance step occurs temperatures around $180 \mathrm{~K}$ and $E_{\omega}=0.32 \mathrm{eV}$ corresponds to even higher temperatures, the voltage dependence at the reverse and moderate forward voltages shown in Fig. 10 cannot be attributed to carrier relaxation, however, it can be attributed to a defect response or the presence of a barrier in the cell structure.

At higher forward biases $(V>0.4 \mathrm{~V})$, the value of the scaled derivative at the peak position rapidly increases with applied voltage for both samples. This increase cannot be explained by the relations listed in Table I. One possible

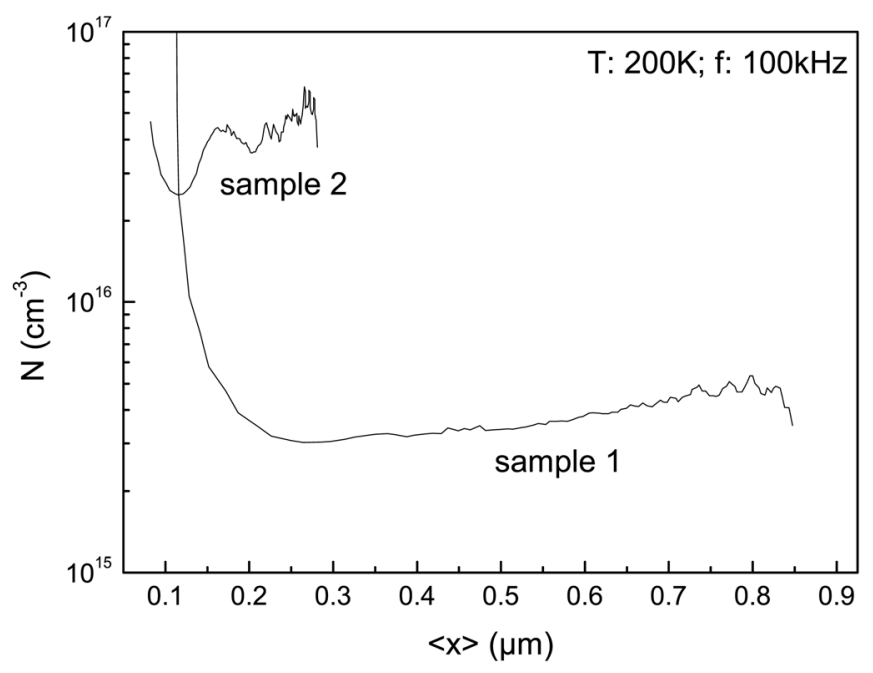

FIG. 11. Apparent doping density profile calculated from capacitance voltage measurements at $200 \mathrm{~K}$ using a $100 \mathrm{kHz}$ test signal. explanation is an increased apparent defect density toward the buffer-absorber interface if the defect level is located close to the valence band (or away from the interface if the defect level is located close to the conduction band). This kind of behavior can be observed when the observed defect is one of the configurations of a metastable defect. ${ }^{18}$ Since the activation energy remains relatively constant, is unlikely that this increase is due to the influence of a back contact barrier. Otherwise, one would expect a large increase in the activation energy according to Eq. (38). There is, however, a small dependence of the activation energy on the applied bias. This could be caused by the bandgap grading which is present in the sample, since this can result in a spatial variation of the defect level energy.

In summary, the capacitance step at lower temperatures in both samples and the capacitance decrease at higher temperatures in sample 2 can be assigned to a bulk defect response. However, a (back) barrier response cannot be totally excluded. The defect density is rather uniform, except for the step at lower frequencies in sample 2, which is nonuniform. At higher forward voltage a second non-uniformity seems to appear.

\section{SUMMARY AND CONCLUSIONS}

The voltage dependence of capacitance frequency measurements has been assessed. A capacitance step due to carrier relaxation, the presence of a defect density, and a (back contact) barrier has been considered. The voltage dependence of the derivative of the capacitance to (the logarithm of) the frequency has been calculated (Table I). These relations can be used to discriminate between the different physical processes leading to capacitance-frequency steps to a limited extent since the voltage dependence for different processes can be similar.

If the capacitance step is due to the presence of a defect density, the assessment of measurements under different dc voltages improves the determination of the defect density, according to the theory by Walter $e t$ al. since the parameters needed in the formulas can be fitted rather than estimated; this fitting procedure additionally provides a confirmation of the used band bending model. Moreover, it is possible to obtain a view of the spatial variation of the defect density while preserving a direct link between the defect level energy and the apparent defect density.

The possibilities and limitations of the calculated relations are checked with numerical simulations on several structures. It is possible to reproduce the input parameters of the simulation with good accuracy through the analysis of $C-f$-simulations by the proposed relations. A nonuniform defect density is easily detected and lower defect densities than through $C-V$-analysis can be analyzed.

The voltage dependence of the admittance spectrum of two thin film $\mathrm{Cu}(\mathrm{In}, \mathrm{Ga}) \mathrm{Se}_{2}$ solar cell devices has been investigated. Even though the presence of a (back contact) barrier cannot be entirely excluded, the capacitance transitions can be assigned to the presence of defect densities. Moreover, the manifestation of a nonspatially uniform defect density was detected. 


\section{ACKNOWLEDGMENTS}

We acknowledge the support of the Research Foundation-Flanders (K.D., FWO Ph.D. fellowship) and of the IWT Project No. 080407.

${ }^{1}$ P. Jackson, D. Hariskos, E. Lotter, S. Paetel, R. Wuerz, R. Menner, W. Wischmann, and M. Powalla, "New world record efficiency for $\mathrm{Cu}(\mathrm{In}, \mathrm{Ga}) \mathrm{Se}_{2}$ thin-film solar cells beyond 20\%," Prog. Photovoltaics (to be published).

${ }^{2}$ D. Schroder, Semiconductor Material and Device Characterization, 2nd ed. (Wiley \& Sons, Toronto, 1998).

${ }^{3}$ J. T. Heath, J. D. Cohen, and W. N. Shafarman, J. Appl. Phys. 95, 1000 (2004).

${ }^{4}$ T. Walter, R. Herberholz, C. Müller, and H. W. Schock, J. Appl. Phys. 80, 4411 (1996).

${ }^{5}$ G. Schumm, Phys. Rev. B 49, 2427 (1994).

${ }^{6}$ M. Igalson and H. W. Schock, J. Appl. Phys. 80, 5765 (1996)

${ }^{7}$ P. Zabierowski, U. Rau, and M. Igalson, Thin Solid Films 387, 147 (2001).

${ }^{8}$ A. Niemegeers, S. Gillis, and M. Burgelman, in Proceedings of the 2nd World Conference on Photovoltaic Energy Conversion, Wien, 1998 (JRC, European Commission), p. 1071.

${ }^{9}$ T. Eisenbarth, T. Unold, R. Caballero, C. A. Kaufmann, and H. W. Schock, J. Appl. Phys. 107, 034509 (2010).

${ }^{10}$ R. Herberholz, M. Igalson, and H. W. Schock, J. Appl. Phys. 83, 318 (1998).

${ }^{11}$ M. Burgelman, P. Nollet, and S. Degrave, Thin Solid Films 361, 527 (2000).
${ }^{12}$ K. Decock, S. Khelifi, and M. Burgelman, Thin Solid Films 519 , 7481-7484 (2011).

${ }^{13}$ J. V. Li, X. N. Li, D. S. Albin, and D. H. Levi, Sol. Energy Mater. Sol. Cells 94, 2073 (2010).

${ }^{14}$ U. Reislöhner, H. Metzner, and C. Ronning, Phys. Rev Lett. 104, 226403 (2010).

${ }^{15}$ S. M. Sze and K. K. Ng, Physics of Semiconductor Devices, 3rd ed. (Wiley \& Sons, New York, 2007).

${ }^{16}$ A. Niemegeers and M. Burgelman, J. Appl. Phys. 81, 2881 (1997).

${ }^{17}$ K. Decock, S. Khelifi, and M. Burgelman, Accuracy of defect distributions measured by bias dependent admittance spectroscopy on thin film solar cells, presented at the 37th IEEE PVSC Area 8, Seattle, 2011.

${ }^{18}$ K. Decock, S. Khelifi, F. Pianezzi, A. N. Tiwari, and M. Burgelman, paper presented at the 37th IEEE PVSC Area2, Seattle, WA, June 19-24 2011.

${ }^{19}$ M. Cwil, M. Igalson, P. Zabierowski, and S. Siebentritt, J. Appl. Phys. 103, 063701 (2008)

${ }^{20}$ L. C. Kimerling, J. Appl. Phys. 45, 1839 (1974).

${ }^{21}$ P. H. Mauk, H. Tavakolian, and J. R. Sites, IEEE Trans. Electron Devices 37, 422 (1990).

${ }^{22}$ J. H. Scofield, Sol. Energy Mater. Sol. Cells 37, 217 (1995).

${ }^{23}$ J. Lauwaert, K. Decock, S. Khelifi, and M. Burgelman, Sol. Energy Mater. Sol. Cells 94, 966 (2010).

${ }^{24}$ K. Decock, J. Lauwaert, and M. Burgelman, Energy Proc. 2, 49 (2010).

${ }^{25}$ S. Buecheler, F. Pianezzi, C. Fella, A. Chirila, K. Decock, M. Burgelman, and A. N. Tiwari, Thin Solid Films 519, 7560-7563 (2011).

${ }^{26}$ A. Chirila, D. Guettler, D. Brémaud, S. Buecheler, R. Verma, S. Seyrling, S. Nishiwaki, S. Haenni, G. Bilger, and A. N. Tiwari, in 2009 34th IEEE Photovoltaic Specialists Conference, (IEEE, New York, 2009), Vols. 1-3, p. 867. 